\title{
A nerve clamp electrode design for indirect stimulation of skeletal muscle
}

Corey J. Hilmas ${ }^{1}$, John W. Scherer ${ }^{2}$, and Patrick T. Williams ${ }^{1}$

${ }^{1}$ Neurobehavioral Toxicology Branch, Analytical Toxicology Division, United States Army Medical Research Institute of Chemical Defense, APG-EA, MD, USA and ${ }^{2}$ Information Management/Technology Office, United States Army Medical Research Institute of Chemical Defense, APG-EA, MD, USA

BioTechniques 49:739-744 (October 2010) doi 10.2144/000113513

Keywords: stimulating electrode; botulinum neurotoxin; skeletal muscle; paralysis

A nerve clamp electrode was developed to indirectly stimulate skeletal muscle innervated by $\alpha$ motor neurons as an alternative to conventional electrodes. The stimulating electrode device consists of a spring coil-activated nerve clamp mounted inside a $1-\mathrm{mL}$ syringe barrel. Supramaximal pulses were generated by a Grass stimulator and delivered to the nerve segment via the nerve clamp electrode. The salient feature of the electrode is its ability to produce muscle contractions indirectly through stimulation of the attached nerve. Indirect muscle stimulation is critical for studying the paralytic actions of presynaptic-acting toxins such as botulinum neurotoxins (BoNT), a potent inhibitor of acetylcholine (ACh) release from $\alpha$ motor neurons. This device enables stimulation of muscle contraction indirectly as opposed to contraction from direct muscle stimulation. The electrode is able to stimulate indirect muscle contraction when tested on ex vivo preparations from rodent phrenic nerve-hemidiaphragm muscle in similar fashion to conventional electrodes. In addition, the electrode stimulated external intercostal nerve-muscle preparations. This was confirmed after applying BoNT serotype A, a potent inhibitor of ACh release, to induce muscle paralysis. Alternative methods, including suction and bipolar loop electrodes, were unsuccessful in stimulating indirect muscle contraction. Therefore, this novel electrode is useful for physiological assessment of nerve agents and presynaptic actions of toxins that cause muscle paralysis. This electrode is useful for stimulating nerve-muscle preparations for which the length of nerve is a concern.

Ex vivo assays to measure muscle paralysis induced by botulinum neurotoxin (BoNT) have been used as functional assays of toxin potency $(1,2$; National Biodefense Analysis and Countermeasures Center Technical Report, TR\#RSRD-04-00140, 2005) and in the detection of antibodies against BoNT (3). Other uses of functional nerveskeletal muscle assays are to evaluate the effectiveness of post-exposure therapeutic candidates against the paralyzing effects of the various BoNT serotypes (4). Ex vivo assays evaluate intact neuromuscular transmission by recording nerve-elicited muscle contractions. While rodent diaphragms are the preferred model system for an ex vivo assay (2,5; National Biodefense Analysis and Countermeasures Center Technical Report, TR\#RSRD-04-00140, 2005), the use of intercostal muscle provides for more preparations to be derived from one animal (6). A single diaphragm provides two preparations, left and right hemidiaphragms, allowing one to serve as an internal control and the other available as an experimental variable. Use of intercostal muscle, with the electrode described, provides additional preparations useful for experiments testing multiple doses and showing proper dose-response functions. An alternative functional technique to quantify BoNT effects on neuromuscular transmission is intracellular microelectrode recordings of BoNT-treated muscle $(7,8)$, where BoNT exposure reduces the frequency of miniature end plate potentials.

Muscle contractility assays are preferred over intracellular microelectrode recording for several reasons. Tension measurements, which indirectly evaluate synaptic activity, are more rapid and provide ensemble averages rather than single cell values (9).

Dissections of phrenic nerve-diaphragm muscle preparations from large rodents provide a sufficiently long nerve to stimulate using common, commercially available electrodes. Typical types include suction-type (10) and bipolar loop-type (5) electrodes. However, dissections of the same nervemuscle preparation from smaller rodents (e.g., mice) often yield small segments of nerve $(<10 \mathrm{~mm})$ due simply to the smaller body size of these animals. Likewise, intercostal muscles-which are major effector organs of respiration and targets for BoNT paralysis-are innervated by nerves too short for suction and bipolar loop electrodes to stimulate contraction. We describe a novel stimulating electrode that overcomes these physiological restrictions and is compatible with commonly used stimulating and recording equipment.

\section{Materials and methods}

Isolation of mouse, guinea

pig, and rabbit tissues

The experimental protocol was approved by the Animal Care and Use Committee at the United States Army Medical Research Institute of Chemical Defense, and all procedures were conducted in accordance with the principles stated in the Guide for the Care and Use of Laboratory Animals (National Research Council, Publication No. 85-23, 1996) and the Animal Welfare Act of 1996 


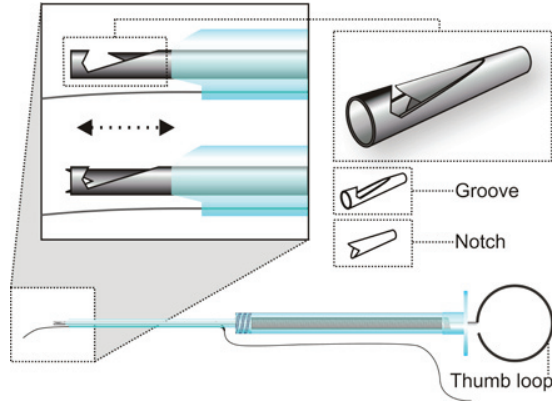

Figure 1. Schematic of the nerve clamp electrode. The nerve clamp electrode uses a spring coil mechanism to hold a segment of nerve in place to stimulate skeletal muscle. Hypodermic tubing, constructed from electrically conducting material, is modified with a notch (region highlighted by dotted lines). The internal guidewire can be pulled back with minimal effort and relax to its normal position via the attached spring to clamp the nerve in place.

(P.L. 89-544), as amended. Healthy adult male mice (Mus musculus; 16-18 g), adult male Hartley guinea pigs (Cavia porcellus; 300-400 g), and adult male New Zealand white rabbits (Oryctolagus cuniculus; 900-1200 g) were purchased from Charles River Laboratories (Wilmington, MA, USA). Rabbits were sedated with acepromazine $(0.5 \mathrm{mg} / \mathrm{kg}$ up to $5 \mathrm{mg} / \mathrm{kg}$ i.m. (11) using a 23-gauge or smaller needle. After sedation, each animal was placed into an induction chamber, and oxygen and isoflurane were administered at $1-2 \mathrm{~L} / \mathrm{min}$ and $3-5 \%$, respectively, until the animal was Mice and guinea pigs were anesthetized with isoflurane, placed in an induction chamber, and euthanized by decapitation.

\section{General methods}

Diaphragm muscle with attached phrenic nerve was dissected from mouse, guinea pig, or rabbit according to methods described elsewhere (12) and incised along the midline recumbent and unresponsive to toe pinch.

into left and right hemidiaphragm preparations. Hemidiaphragm muscle-nerve preparations were mounted in a vertical twitch bath (6- or 60-mL capacity). Muscle tendon was fixed to a stainless steel hook anchored to the bottom of a temperature-controlled tissue organ bath (Radnoti Glass Technology, Monrovia, CA, USA) at $37^{\circ} \mathrm{C}$ and tied to a force-displacement transducer using $4 / 0$ silk thread on the opposite end. A series of three stimulating electrodes were placed on each attached nerve and stimulated with 100-us supramaximal pulses (7.5 V) using an S88 Grass stimulator (Grass Instruments, Warwick, RI, USA). Stimulation of the nerve using the suction electrode was always followed by a bipolar loop and finally the nerve clamp electrode, in that order.

\section{Recording of twitch tensions}

The bath contained Tyrode's solution of the following composition: $137 \mathrm{mM} \mathrm{NaCl}$, $2.7 \mathrm{mM} \mathrm{KCl}, 11.9 \mathrm{mM} \mathrm{NaHCO}, 0.3 \mathrm{mM}$ $\mathrm{NaH}_{2} \mathrm{PO}_{4}, 0.5 \mathrm{mM} \mathrm{MgCl}, 1.8 \mathrm{mM}$ $\mathrm{CaCl}_{2}$, and $5.6 \mathrm{mM}$ glucose. The $\mathrm{pH}$ was maintained at 7.3 by bubbling with a gas mixture of $95 \% \mathrm{O}_{2}$ and $5 \% \mathrm{CO}_{2}$. Muscles were stimulated indirectly via the nerve clamp, bipolar loop-type, or suction-type electrodes using an S88 Grass stimulator. Muscle tension was recorded with Grass FT 03 isometric force-displacement transducers (Grass Instruments, Warwick, RI, USA), displayed on a Gould TA240 EasyGraf chart recorder (Gould Instruments, Valley View, $\mathrm{OH}, \mathrm{USA}$ ), and digitized using pCLAMP software (Molecular Devices, Sunnyvale, CA, USA). A bath concentration of $1 \mathrm{nM}$ BoNT serotype A (BoNT/A; Metabiologics, Madison, WI, USA) results in complete muscle paralysis of mouse, guinea pig, and rabbit muscle in $\sim 1 \mathrm{~h}$. Application of BoNT/A does not decrease force tension in muscles directly stimulated by electrodes, because BoNT/A acts presynaptically.

Table 1. Peak amplitude and decay time of twitch tensions recorded from the various stimulating electrodes.

\begin{tabular}{|c|cccc|}
\multirow{2}{*}{$\begin{array}{c}\text { Type of } \\
\text { stimulating } \\
\text { electrode }\end{array}$} & \multicolumn{4}{|c|}{ Average peak amplitude (g) } \\
\cline { 2 - 5 } & $\begin{array}{c}\text { Mouse } \\
\text { hemidiaphragm }\end{array}$ & $\begin{array}{c}\text { Guinea pig } \\
\text { hemidiaphragm }\end{array}$ & $\begin{array}{c}\text { Rabbit } \\
\text { hemidiaphragm }\end{array}$ & $\begin{array}{c}\text { Rabbit } \\
\text { intercostal }\end{array}$ \\
\cline { 2 - 5 } Nerve clamp electrode & $4.86 \pm 0.97$ & $16.83 \pm 6.35$ & $48.20 \pm 14.18$ & $3.38 \pm 1.12$ \\
\hline Bipolar loop electrode & $4.43 \pm 0.71$ & $17.74 \pm 8.25$ & $54.05 \pm 7.87$ & - \\
\hline Suction electrode & $4.12 \pm 1.58$ & - & - & - \\
\hline \multirow{4}{*}{$\begin{array}{c}\text { Type of } \\
\text { stimulating } \\
\text { electrode }\end{array}$} & \multicolumn{4}{|c}{ Decay time (ms) } \\
\cline { 2 - 5 } & Mouse & Species and muscle type & Rabbit \\
\hline Nerve clamp electrode & $22.11 \pm 5.03$ & $52.93 \pm 14.55$ & $157.21 \pm 42.10$ & $169.08 \pm 40.64$ \\
\hline Bipolar loop electrode & $23.78 \pm 6.09$ & $54.82 \pm 16.15$ & $136.05 \pm 33.01$ & - \\
\hline Suction electrode & $19.30 \pm 4.10$ & - & Rabbit \\
\hline
\end{tabular}

For each muscle preparation (hemidiaphragm or intercostals), no significant differences in the recording tensions between any of the electrode types were determined $(P>0.05)$.

\section{Electrode design}

A schematic drawing of the nerve clamp electrode is illustrated in Figure 1. The assembled device, built within a 1-mL syringe, contains an adjustable spring coil at one end and the nerve clamp-stimulating portion at the other. The electrode was constructed from a metal hypodermic tubing and guidewire assembly (Small Parts, Miami Lakes, FL, USA). A groove was filed into the end of the guidewire (0.046-in diameter) to clamp the nerve in place. The guidewire fits inside a sleeve of hypodermic tubing 16 gauge; outer diameter $0.065 \mathrm{in}$; inner diameter $0.047 \mathrm{in}$ ]. The tubing was modified at one end with a notch for a small segment of nerve to pass inside. The sliding action of the guidewire allows nerves to be held in contact with the hypodermic tubing, which is the stimulation portion of the electrode.

The internal guidewire was made adjustable and placed under tension at rest by cementing it to a spring coil at the proximal end of the electrode. Both spring coil and guidewire were placed inside a 1-mL tuberculin syringe, and the spring coil was cemented to the syringe at a point nearest the narrow syringe opening. The internal guidewire was bent and shrink-wrapped with plastic for fine finger/thumb adjustment.

Instructions for the assembly of this electrode are described and illustrated in Figure 2. In Step 1, a notch was cut into the hypodermic tubing at one end using a fine file, and a sheath of polyethylene tubing was placed tightly over the hypodermic tubing as insulation. A platinum/iridium stimulating wire $(0.25$-mm diameter) was soldered to the exposed hypodermic tubing. This assembly from Step 1 is positioned inside of a second, larger-diameter polyethylene tubing along with a second platinum/iridium wire, which serves as the ground (see Step 2).

The internal guidewire was prepared at both ends in Step 3. A groove was filed at the nerve clamp end, and the wire was bent into a loop at the other. The spring coil was placed inside of the $1.0-\mathrm{mL}$ syringe barrel and cemented inside. The guide was inserted inside the spring and syringe barrel and cemented to the spring coil. The assembled stimulating/ground portion from Step 2 was inserted over the guidewire into the syringe barrel. The hypodermic tubing was cemented with epoxy to the syringe tip. The platinum/ iridium stimulating and ground wires were subsequently connected to a Bayonet-NeillConcelman (BNC) cable to be used with the S88 Grass stimulator.

The cut end of a motor nerve innervating a muscle can be clamped and fixed in space using the guidewire assembly in the following manner: when the spring is pulled back, the electrode can be positioned such that any 

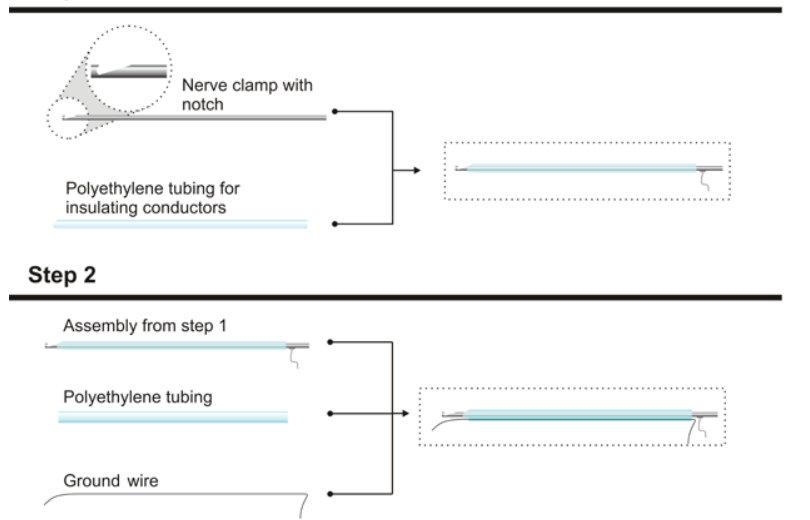

Step 3

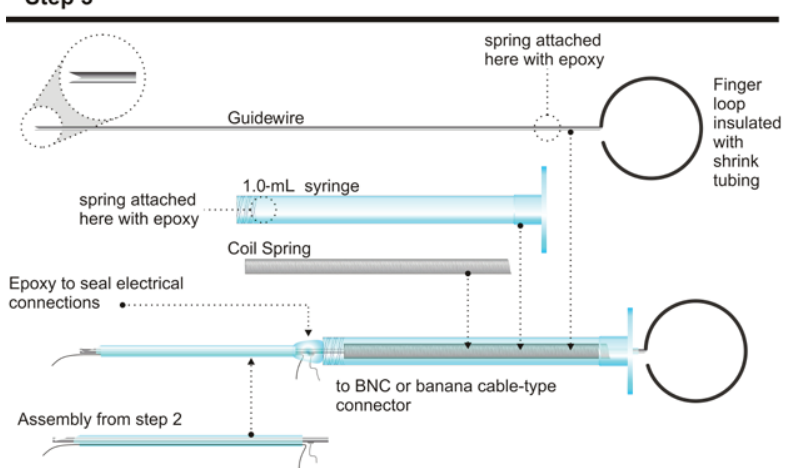

Figure 2. Three-step assembly of the nerve clamp electrode. (Step 1) The electrode is assembled using a hypodermic tube and guidewire assembly, in which a notch on the tubing and groove in the guidewire are filed into the metal pieces, respectively. Polyethylene tubing covers the tube as insulation. (Step 2) A stimulating wire is soldered to the metal tubing; the ground wire is fixed in place with epoxy cement and sheathed on the outside with another layer of polyethylene tubing for insulation. (Step 3) The tubing and guidewire assembly are passed through a 1-mL syringe and cemented to a spring coil inside. The guidewire is made to move freely while pulling back on the insulated thumb ring connected to the spring coil.

segment length of nerve can be fixed in space against the hypodermic tubing. Release of the spring coil will return the guidewire to its normal position inside the hypodermic tubing and clamp the nerve in place. The stimulator generates supramaximal pulses, causing the clamped nerve to generate muscle contractions, provided the nerve was not damaged during dissection. The extent of muscle contraction can be recorded using an isometric force transducer that converts the force of contraction into a digitized wave form to be analyzed later on pCLAMP software. The tensions can be recorded directly onto a chart recorded as analog waveforms. This method of stimulation enables the study of substances that act either presynaptically in the nerve, such as botulinum toxin, or act at the level of the neuromuscular junction, such as nerve agents. The major advantage of the described electrode is its ability to indirectly stimulate any skeletal muscle, regardless of nerve length, for the measurement of muscle force tension using tissue organ baths.

Data analysis

Muscle contraction data generated from suction-type and bipolar loop-type electrodes were compared with muscle twitch data obtained using the nerve clamp electrode. Peak amplitude and $10-90 \%$ decay time from twitch tension recordings were calculated for all three electrodes. To prevent direct stimulation of the hemidiaphragm muscle preparation, stimulation intensity was preset to a maximum of $7.5 \mathrm{~V}$. Muscle paralysis was subsequently recorded in the presence of BoNT/A. Twitch tension peak amplitude and $10-90 \%$ decay times were compared using a two-factor ANOVA. Each peak amplitude and 10-90\% decay time reported reflect mean \pm SD of twitch recordings from 7-11 separate muscles.

\section{Results and discussion}

Hemidiaphragm muscle twitch tension amplitudes on the order of $0.5-6 \mathrm{~g}$ for mouse diaphragm, 10-40gforguineapigdiaphragm, $20-60 \mathrm{~g}$ for rabbit diaphragm, and 4-18 g for rabbit external intercostal muscle were recorded following stimulation of the nerve with the described nerve clamp-stimulating electrode device (see Figure 1). Representative control twitch tension recordings are shown (Figure 3A) before BoNT/A application. The shape, twitch amplitudes, and time course of decay were very similar to results obtained using bipolar loop and suction electrodes for mouse, guinea pig, and rabbit diaphragm. These values have been summarized in Table 1. Due to the short length of motor nerve innervating rabbit external intercostal muscle, it was not possible to stimulate this indirectly using bipolar loop (data not shown) or suction electrodes (Figure 3B). Control experiments for all muscles showed stable twitch tensions during the time course of study; tensions decreased to only $70 \%$ of their initial value after $\sim 8.5 \mathrm{~h}$ in the tissue bath (data not shown). None of the twitch tension amplitudes from control experiments decreased to $50 \%$ of their initial value after almost $9 \mathrm{~h}$ in the tissue bath. The first twitch tension recordings following application of BoNT/A to the tissue bath showed decrease in the twitch tension amplitude (Figure 4A), suggesting muscle contraction was indeed the result of indirect muscle stimulation through the nerve. Muscle preparations in the absence of BoNT/A maintained their tension throughout the duration of the recording period (data not shown). Traces shown in Figure $4 \mathrm{~A}$ represent 10 -min intervals. If the muscle was stimulated directly, BoNT/A would have no effect on the muscle contraction amplitude. Figure $4 \mathrm{~B}$ shows the dose-dependent effect of BoNT/A ( $1 \mathrm{pM}$ and $1 \mathrm{nM})$ to excised rabbit external intercostal muscle as a function of time. Intercostal hemidiaphragm muscle preparations in two separate experiments were stimulated indirectly through the external intercostal nerve using the described nerve clamp electrode. All test concentrations of BoNT/A resulted in complete paralysis of the muscle during the same time course of study. BoNT/A exhibited dose dependence, where the higher BoNT/A test concentration $(1 \mathrm{nM})$ gave the shortest times to reach $50 \%$ inhibition, and a smaller concentration $(1 \mathrm{pM})$ resulted in a longer time to $50 \%$ inhibition and subsequently complete muscle paralysis. This is in agreement with many previous findings using bipolar loop stimulating electrodes.

Bipolar loop electrodes are more advantageous than suction-type electrodes as they allow stimulation of larger-diameter nerve segments, typically with excess adipose tissue adhered to the axon tract. However, they are also not without their limitations. The nerve must be long enough $(>15 \mathrm{~mm})$ to tie a ligature around the proximal end in order to thread it through the platinum wire loops. Likewise, suction electrodes require sufficient length to apply negative pressure to maintain the nerve inside the tubing shaft of the electrode. The negative pressure created by suction electrodes can cause the 
A

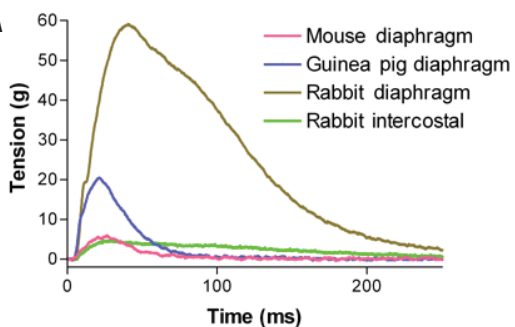

B

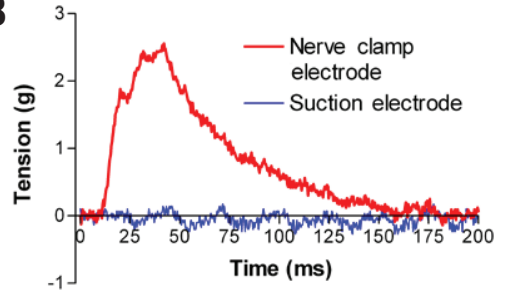

Figure 3. Indirect muscle twitch tension recordings stimulated using the nerve clamp electrode. (A) Representative traces of twitch tensions recorded from hemidiaphragm and intercostal muscles from mouse, guinea pigs, and rabbits in the absence of BoNT using the described nerve clamp electrode. (B) Twitch tension recordings from indirect stimulation of rabbit external intercostal muscle. Dissections of rabbit intercostal muscle for physiological assays yielded preparations with intercostal nerve of insufficient length to be held in contact with conventional electrodes for measurement of contractile force. A suction electrode failed to generate twitch force tension in the intercostal muscle preparation as did a bipolar loop electrode in the same preparation (data not shown); the described nerve clamp electrode did subsequently stimulate the intercostal nerve. Tensions were elicited by supramaximal stimulation ( $7 \mathrm{~V}$ ) of the nerve every $30 \mathrm{~s}$ in a vertical twitch bath.

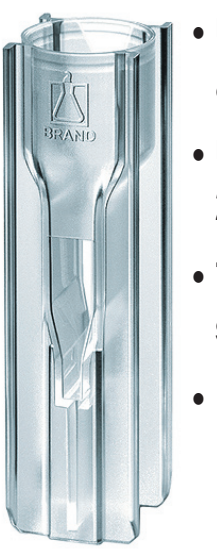

\section{- UV-transparent and disposable}

- Use between 220-900nm

- $70 \mu \mathrm{L}$ minimum sample volume

-10mm lightpath

- Replace quartz cuvettes

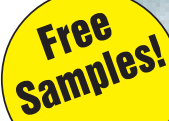
for DNA, RNA and proteins

\section{- Eliminate risk of} cross-contamination from cuvette re-use

- Convenient \& economical: no more cuvette washing!

- Simplify sample storage: round caps hold tight

\section{- Available individually} wrapped, DNA-, DNase-, \& RNase-free

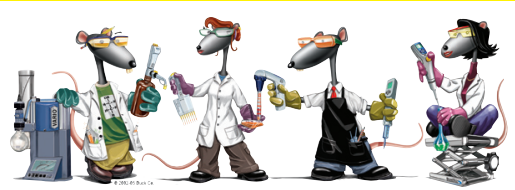

Lab Rats Trust BrandTech!

BRANDTECH SCIENTIFIC, INC.
A

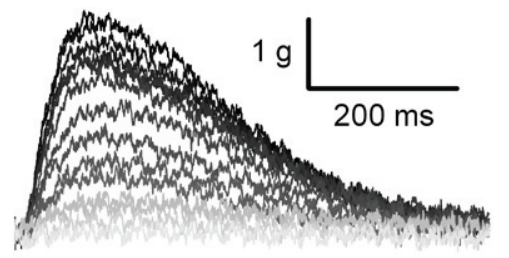

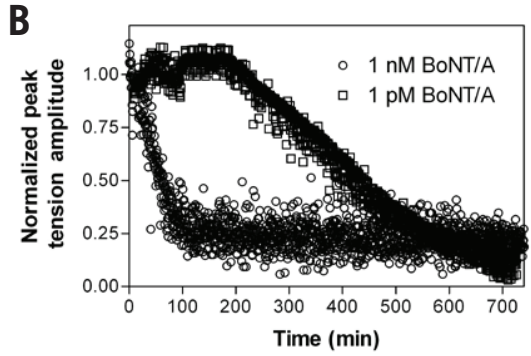

Figure 4. Stimulation and paralysis of rabbit intercostal muscle by BoNT/A (1 nM). (A) Each trace represents the twitch tension elicited by indirect stimulation of rabbit intercostal muscle in a single experiment by the described nerve clamp electrode. BoNT/A was added just prior to acquisition of the first twitch tension. The first twitch tension, generated immediately after BoNT application to the tissue bath, is always the largest in peak amplitude. Twitch tension was recorded at 10-min intervals until complete paralysis (no muscle tension recorded following muscle stimulation). (B) Normalized twitch tension amplitudes decrease as a function of time following BoNT/A application in a dose-dependent manner. Normalized peak amplitudes were generated by dividing twitch tension amplitudes by the average twitch tension amplitude of control traces prior to addition of BoNT/A. Each curve represents the time to paralysis from two separate experiments. Toxin was added at 0 min. Control twitch tension amplitudes (no BoNT/A added) maintain their magnitude throughout the recording period of the study (not shown). Tensions were elicited by supramaximal stimulation $(7.5 \mathrm{~V})$ of the nerve every $30 \mathrm{~s}$ in a vertical twitch bath.

nerve to pull away from the muscle, leading to denervation.

The intent of this investigation was to design and construct a unique electrode capable of eliciting muscle contractions indirectly, regardless of anatomical constraints. Typically, the common peroneal nerve-extensor digitorum longus, tibial nerve-soleus, and phrenic nervehemidiaphragm muscle preparations are used to indirectly stimulate muscle because they meet this nerve length requirement. This nerve clamp-stimulating electrode possesses several advantages over currently available stimulating electrodes. The described nerve clamp electrode performs similarly to commonly used electrodes, and data obtained from its use are consistent with data obtained from other electrodes. Peak amplitude of the muscle twitch tension as well as decay time did not significantly differ among all three electrodes tested. It also is not restricted by the physical characteristics of the nerve. Any nerve length or thickness can be securely and reliably contacted by the nerve clamp without directly contacting the muscle itself. It represents an improvement over commonly used electrodes, as it is able to indirectly stimulate muscles in preparations where commonly used electrodes fail, and is compatible with existing commonly used physiology laboratory stimulation and data recording hardware.

\section{Acknowledgments}

The opinions, interpretations, conclusions, and recommendations are those of the author(s) and are not necessarily endorsed by the US Army or the Department of Defense.

\section{Competing interests}

The authors declare no competing interests. 


\section{Don't Be Threatened by Uncertainty}

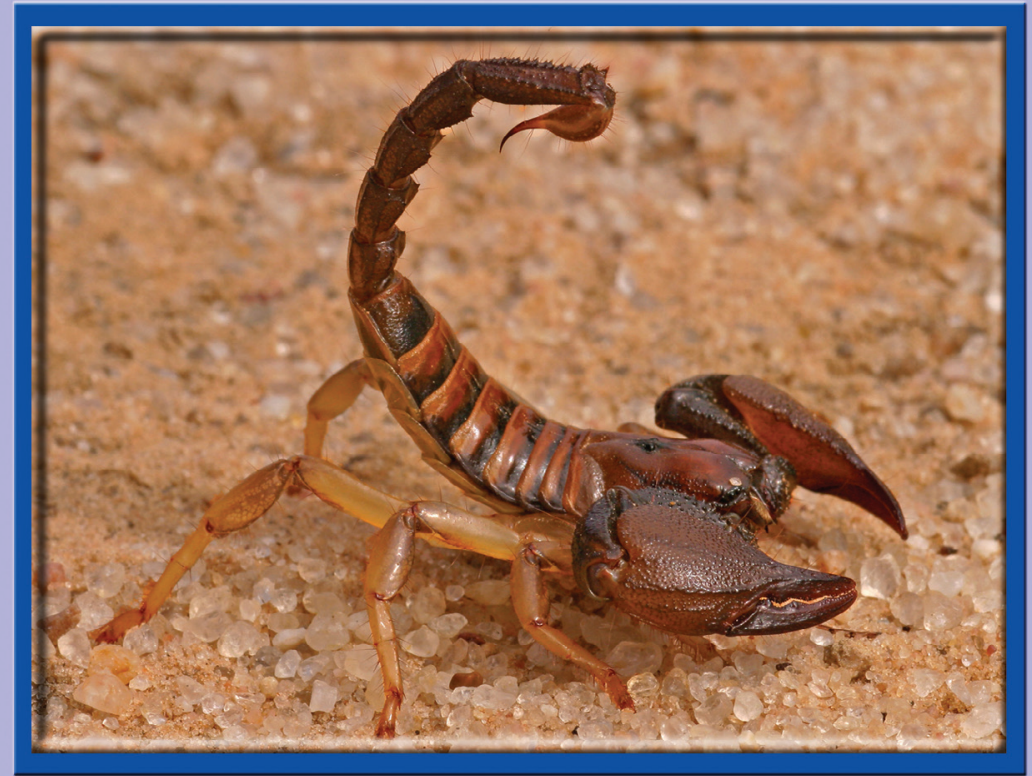

\section{Adding toxicogenomics to your standard} toxicology programs can help with difficult decisions in your drug development programs

\author{
$\star$ Assay Development \\ $\star$ Nucleic Acid Purification \\ $\star$ Genotyping \\ $\downarrow$ Microarray Technology \\ $\star$ qRT-PCR \\ Toxicogenomics \\ $\forall$ Bioinformatics \\ $\star$ Tumor Profiling \\ - Sample Management \\ $\star$ Biostorage
}

\section{THE Pharmacogenomics Company}

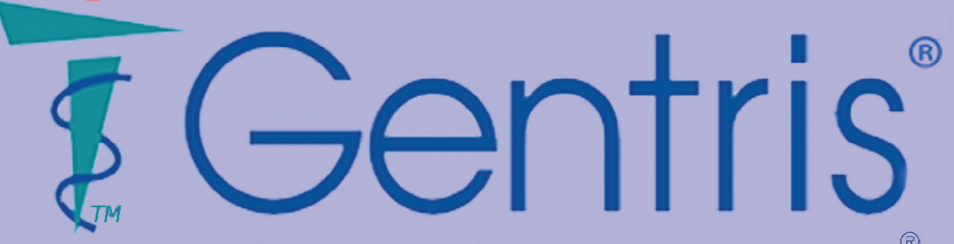

Revolutionizing Medicine Through Pharmacogenomics

\section{References}

1.Adler, M., H.A. Manley, A.L. Purcell, S.S. Deshpande, T.A. Hamilton, R.K. Kan, G. Oyler, O. Lockridge, et al. 2004. Reduced acetylcholine receptor density, morphological remodeling, and butyrylcholinesterase activity can sustain muscle function in acetylcholinesterase knockout mice. Muscle Nerve 30:317-327.

2.Hilmas, C.J. and P.T. Williams. 2006. A comparative study of skeletal muscle sensitivity to botulinum neurotoxin serotypes A, B, and $E$ in three mammalian species. Proceedings of the 15th Bioscience Review. Hunt Valley, MD, 4-9 June 2006.

3. Sesardic, D., R.G. Jones, T. Leung, T. Alsop, and R. Tierney. 2004. Detection of antibodies against botulinum toxins. Mov. Disord. 19(Suppl 8):S85-S91.

4.Deshpande, S.S., R.E. Sheridan, and M. Adler. 1995. A study of zinc-dependent metalloendopeptidase inhibitors as pharmacological antagonists in botulinum neurotoxin poisoning. Toxicon 33:551-557.

5.Sheridan R.E. and S.S. Deshpande. 1995. Interactions between heavy metal chelators and botulinum neurotoxins at the mouse neuromuscular junction. Toxicon 33:539-549.

6. Straughan, D. 2006. Progress in applying the three Rs to the potency testing of boutlinum toxin type A. Altern. Lab. Anim. 34:305-313.

7.Tse, C.K., D. Wray, J. Melling, and J.O. Dolly. 1986. Actions of beta-bungarotoxin on spontaneous release of transmitter at muscle end-plates treated with botulinum toxin. Toxicon 24:123-130.

8. Coffield, J.A., N. Bakry, R.D. Zhang, J. Carlson, L.G. Gomella, and L.L. Simpson. 1997. In vitro characterization of botulinum toxin types A, C and D action on human tissues: combined electrophysiologic, pharmacologic and molecular biologic approaches. J. Pharmacol. Exp. Ther. 280:1489-1498.

9. Eckert, R. and D. Randall. 1978. Muscle and movement, p. 279-317. In Animal Physiology. Freeman Publishing, San Francisco.

10.Easton, D.M. 1996. Muscle chamber with strain gauge adapted for isotonic/ isometric recording. Am. J. Physiol. 270:S29S39.

11.Hawk, C.T. and S.L. Leary. 1999. Formulary for Laboratory Animals. Iowa State University Press, Ames, IA.

12.Deshpande, S.S., E.X. Albuquerque, and L. Guth. 1976. Neurotrophic regulation of prejunctional and postjunctional membrane at the mammalian motor endplate. Exp. Neurol. 53:151-165.

Received 29 July 2009; accepted 4 August 2010.

Address correspondence to Patrick Williams, Neurobehavioral Toxicology Branch, Analytical Toxicology Division, U.S. Army Medical Research Institute of Chemical Defense, 3100 Ricketts Point Road, Aberdeen Proving Ground, MD 21010-5400, USA. e-mail: patrick.williams23@us.army.mil 